DOI: https://doi.org/10.46296/yc.v5i9edespsep.0101

\title{
ESTUDIO DEL PERFIL SOCIO-ECONÓMICO DE ESTUDIANTES DE PRIMERO Y SEGUNDO NIVEL DE LA ESPAM MFL
}

\section{STUDY OF THE SOCIO-ECONOMIC PROFILE OF FIRST AND SECOND LEVEL STUDENTS OF THE ESPAM MFL}

\author{
Ormaza-Murillo María Piedad ${ }^{1}$; Zamora-Cusme Maryuri Alexandra 2; Macías-Muñoz
} Gabriela Thalía ${ }^{3}$; Vélez-Zambrano Erika Cecibel ${ }^{4}$

${ }^{1}$ Escuela Superior Politécnica Agropecuaria de Manabí. PhD en Ciencias Técnicas. Vicerrectora Académica e Investigación. Calceta, Ecuador. Correo: mormaza@espam.edu.ec. ORCID ID: https://orcid.org/0000-0002-6558-5826

${ }^{2}$ Escuela Superior Politécnica Agropecuaria de Manabí. Doctora en Ciencias Contables y Empresariales. Calceta, Ecuador. Correo: mzamora@espam.edu.ec. ORCID ID: https://orcid.org/0000-0001-9768-3212

${ }^{3}$ Ingeniera comercial mención en Administración Pública. Calceta. Ecuador. Correo: gmacias549@gmail.com. ORCID ID: https://orcid.org/0000-0003-0763-0390

${ }^{4}$ Ingeniera comercial mención en Administración Pública. Calceta, Ecuador. Correo: cecineidy@gmail.com. ORCID ID: https://orcid.org/0000-0003-2206-5713

\section{Resumen}

Objetivo. Realizar un levantamiento del perfil socio-económico de los estudiantes que ingresan a las carreras de grado de la Escuela Superior Politécnica Agropecuaria de Manabí Manuel Félix López. Método. La tecnica utilizada fue de campo y documental, así como los métodos: deductivo e inductivo y analítico-sintético. Se empleó como instrumento de recolección de datos la encuesta y la entrevista, la encuesta fue aplicada a 924 estudiantes y la entrevista a los ocho Directores de Carreras. Utilizando el programa estadístico SPSS como procesador de datos. Resultados. Los hallazgos encontrados revelan que para acceder a la universidad el $40,9 \%$ de los estudiantes realizaron cambio de residencia y alrededor del $10 \%$ de la población estudiantil tienen hijos que mantener.

Palabras claves: Perfil socio-económico, plan estratégico, permanencia, deserción, rendimiento académico.

\begin{abstract}
Objective. Research is to carry out a survey of the socio-economic profile of the students who enter the undergraduate careers of the Escuela Superior Politécnica Agropecuaria de Manabí Manuel Félix López. Method. The technique used was field and documentary, as well as the methods: deductive and inductive and analytical-synthetic. Was used as an data collection instrument the survey and the interview, the survey was applied to 924 students and the interview to the eight directors of the career. Using the statistical program SPSS as the data processor. Results. The findings found reveal that in order to access the university, $40.9 \%$ of the students made a change of residence and around $10 \%$ of the student population have children to support.
\end{abstract}

Keywords: Socio-economic profile, strategic plan, permanence, desertion, performance.

Información del manuscrito:

Fecha de recepción: 02 de julio de 2021.

Fecha de aceptación: 09 de septiembre de 2021.

Fecha de publicación: 17 de septiembre de 2021. 


\section{Introducción}

La educación, es el punto de partida para el desarrollo económico y social debido a que proporciona los mecanismos necesarios para ampliar y adquirir nuevos conocimientos que les permiten a las personas ser altamente competitivas y productivas en el mercado laboral. En los últimos años, ha adquirido mayor pertinencia como eje clave el paradigma de la igualdad, que ha favorecido el crecimiento económico debido a la especialización, además de aumentar la eficiencia y eficacia en diversas áreas como: salud, tecnología, agricultura, entre otros. La Comisión Económica para América Latina y el Caribe (CEPAL, 2019), considera que: "La educación es un eslabón que contribuye a conciliar el crecimiento, la equidad y la participación en la sociedad".

El Estado ecuatoriano tiene el deber constitucional de garantizar los medios y facilitar el acceso a la educación superior. La Constitución de la República del Ecuador (2008) en su artículo 26, atribuye gran importancia a la educación universitaria fundamentalmente porque es considerada un sector prioritario en las políticas públicas y estratégicas en la formación de capital humano necesarios para el crecimiento y desarrollo económico y social en el Ecuador. Dada la importancia de la educación superior, en los últimos años el gobierno ecuatoriano decidió realizar cambios significativos, que tuvieron como objetivo principal mejorar la calidad de enseñanza-aprendizaje para elevar los niveles de rendimiento académico, permanencia y altas tasas de titulación.

"El ingreso a las instituciones públicas de educación superior se regulará a través de un sistema de nivelación y admisión, definido en la Ley. La gratuidad se vinculará a la responsabilidad académica de los estudiantes" (Art. 356 Asamblea Nacional de la Republica del Ecuador, 2008, p. 110). Sin embargo, pese a que la Constitución expresa que la educación superior es gratuita, hay insumos de educación que corren por cuenta del estudiante, siendo los problemas socio-económicos una de las causas que más dificultan el acceso a la educación superior; en este sentido, el Instituto Nacional de Evaluación 
Educativa (INEVAL, 2020), menciona que, uno de los principales factores determinantes en el rendimiento académico, está relacionado con aspectos del índice socioeconómico.

Según investigaciones realizadas por (Coschiza et al., 2016) demuestran que al menos uno de cada tres estudiantes trabaja mientras cursa sus estudios universitarios y uno de cada siete estudiantes busca trabajo pero no lo encuentra, pues de las contribuciones económicas, la principal fuente de financiamiento de los estudios es la familiar $(70 \%$ de los alumnos), la otra parte, a través de becas, planes sociales $u$ otras fuentes mucho menos relevantes; y respecto al nivel de educación alcanzado por los padres, predominan quienes no han completado los estudios secundarios.

En este contexto, se puede argüir que el bienestar social y el crecimiento económico del país se ve afectado, como consecuencia de la deserción estudiantil y de la escaza preparación por la preocupación constante de generar ingresos por parte de los estudiantes, lo que a su vez dificulta enfrentar las demandas del mundo laboral y ser más competitivo. La deserción universitaria se ha ido incrementando en los últimos años, con mayor notoriedad en los primeros semestres; las principales causas obedecen a la mala elección de la carrera, falta de compromiso, problemas familiares, y bajos niveles económicos, lo que a su vez trae como consecuencia un bajo rendimiento académico y abandono de la universidad.

La deserción estudiantil no solo afecta al estudiante en su desarrollo personal y familiar, también lo hace con la institución de educación superior, ya que incide al cumplimiento de sus objetivos $y$ metas institucionales; y la sociedad misma retrasando los avances socioeconómicos y tecnológicos del país.

Por lo anteriormente expuesto, es necesario conocer la realidad social y económica que tiene cada estudiante, buscando las estrategias idóneas para el mejoramiento no solo de su rendimiento académico, sino también del proceso de adaptación a la universidad, y evitar - reducir los altos índices de deserción estudiantil e incremento de la tasa de titulación en las 
carreras de grado de la ESPAM MFL.

La Escuela Superior Politécnica Agropecuaria de Manabí Manuel Félix López en su modelo educativo (2016) señala que para hacer frente a las tensiones y problemas del Plan Nacional de Desarrollo Toda una Vida 2017-2021 en la zona 4, Hábitat Sustentable, Eje: educación, que las unidades académicas de investigación y de vinculación están empeñadas en fomentar la formación del talento humano en los diferentes niveles educativos y de profesionalización, incrementar el acceso de la población a la educación superior, asimismo, trabajar por la universalización de la educación y diálogo de saberes con derechos, equidad de género, enfoque ambiental, intergeneracional, intercultural, étnico e inclusión de todos en pos de incrementar la calidad de la educación superior en la zona 4.

En referencia de lo antes mencionado, la ESPAM MFL recibe aproximadamente 500 estudiantes cada semestre, de los cuales según reportes emitidos por la institución educativa en los últimos semestres, se ha evidenciado un porcentaje de deserción estudiantil en los primeros semestres; es importante destacar, que la mayoría de los estudiantes llegan de distintos lugares de la provincia de Manabí, de otras provincias del país y un elevado porcentaje de ellos viven en el cantón Bolívar.

Los estudiantes forman parte imprescindible de la universidad, por eso es necesario conocer y estudiar su situación socio-económica, la base económica de la familia, medios económicos para su sustento, continuidad y permanencia estudiantil, tomando en consideración el lugar de procedencia, ya que debido a esto incurren en costos de arriendo, alimentación, viáticos y vestimenta. El estudio del perfil socio-económico facilitará la sistematización de información, que servirá de base para futuras investigaciones en las que se desarrollen estrategias que contribuyan al incremento del rendimiento académico, mejoramiento de la tasa de retención, disminuir la deserción e incrementar la tasa de titulación en las carreras de grado de la ESPAM MFL. 
Por tal motivo, ésta investigación tiene como objetivo realizar un levantamiento de datos socioeconómicos de los estudiantes que ingresan a las carreras de grado de la ESPAM MFL y establecer parámetros de su realidad social y económica.

\section{Método}

La investigación se realizó en la Escuela Superior Politécnica Agropecuaria de Manabí "Manuel Félix López", ubicada en el cantón Bolívar, ciudad de Calceta, sector el Limón. El estudio del perfil socioeconómico fue realizado sobre una muestra de 924 estudiantes provenientes de una población de 1113 del primer y segundo nivel de las carreras de grado de la ESPAM MFL, durante el período académico Abril/2019-Marzo/2020. Se emplea el método inductivo pues según (Hernández et al., 2014), este se orienta hacia la investigación de campo, con la utilización de herramientas como la encuesta, aplicada a los estudiantes de primero y segundo nivel y la entrevista a los directores de carrera, este método permite mantener contacto con el objeto de estudio, medio ideal para recolectar los datos necesarios para el levantamiento del perfil socioeconómico de los estudiantes que ingresaron a las carreras de la ESPAM "MFL". La aplicación del método deductivo permite según (Palencia Avendaño, 2012) tomar como punto de partida una teoría general extrayendo explicaciones muy puntuales de una realidad, por medio de la lógica y el sentido común, la aplicación del mismo, fue fundamental para aclarar el concepto del perfil socio-económico, definiéndolo a través del marco teórico, para luego, a partir de las encuestas aplicadas, ir desmembrando las características fundamentales, proporcionando información útil para la investigación.

Instrumentos de recolección de datos

Las técnicas de investigación son los medios que hacen operativos a los métodos, las cuales fueron utilizadas como instrumentos de recolección y análisis, de los datos obtenidos por medio de la encuesta y entrevista (Palencia Avendaño, 2012). Se utilizó como técnica de investigación la encuesta siendo fundamental en recopilación de datos como: datos generales, opiniones, sugerencias 0 
respuestas que se proporcionen a preguntas formuladas sobre los diversos indicadores que se pretenden explorar. Esta se aplicó por medio de un cuestionario de preguntas a los estudiantes de los primeros y segundos niveles del período académico Abril/2019Marzo/2020 de la ESPAM MFL.

Descripción de la muestra

La entrevista se realizó de forma directa y estandarizada, con base a un cuestionario de preguntas abiertas al personal directivo de las ocho carreras de la ESPAM MFL, con el fin de obtener información de los estudiantes que ingresaron a las carreras de grado en la universidad, destacando aspectos socioeconómicos y la repercusión que tiene dentro del rendimiento académico de los estudiantes y la importancia de incentivarlos por medio de becas y charlas motivacionales.

Los tipos de investigación son las distintas formas de indagar (Hernández et al., 2014), en el presente estudio se aplicó la investigación documental y de revisión bibliográfica, así como la investigación de campo, las cuales se basan en la búsqueda de información y hechos con la finalidad de conocer una verdad esencial para complementar y sustentar teóricamente la investigación.

Finalmente se emplea el programa estadístico SPSS (Statistical Package for the Social Sciences) versión 2.3 que permite ingresar una base de datos con sus respectivas opciones de variables, para luego diseñar cuadros estadísticos, gráficos de porcentajes y variables de las preguntas.

\section{Resultados}

Posterior al análisis de los datos se obtuvieron los siguientes resultados que fueron desagregados por datos generales, sociales y económicos, para facilitar el análisis y comprensión de la situación socioeconómica de los estudiantes. 




Fig. 1. Datos generales. Elaborado por el autor (2020). Nota: MV: Medicina Veterinaria; $A D$ : Administración; IA: Ingeniería Ambiental; Agl: Agroindustrias; Cp: Computación; AP: Administración Pública; Ag Agrícola; Tr: Turismo.

En la figura 1, se exhiben los datos generales derivados de la pregunta relativa al número de estudiantes por carrera, el $20.5 \%$ pertenecen a Medicina Veterinaria, seguido de Administración de Empresas con el 15.6\%, Ingeniería Ambiental 13.6\%, Agroindustria 12.3\%, Computación 11.8\%, Administración Pública $11.3 \%$, Agrícola $8.3 \%$, y por último Turismo con el 6.6\%, siendo la carrera de Medicina Veterinaria que cuenta con un mayor número de estudiantes.

En relación al total de estudiantes de primer y segundo nivel, es importante considerar que la cantidad de estudiantes que cursan el segundo nivel es inferior a los de primero con un $31 \%$, evidenciando que alrededor de la mitad, no aprueban el primer nivel debido a que muchos desertan, repiten materias e incluso se retiran de la carrera universitaria. En este sentido, (Peña, 2017) expone que:

La deserción a partir de la experiencia universitaria, influye directamente en los estudiantes al menos en los primeros niveles de los programas educativos. Los casos sobre experiencia universitaria que desembocan en una deserción universitaria son varios entre los cuales se destacan: la expectativa académica relacionado a la carga horaria universitaria, los nuevos deberes que adquiere en la universidad, el ajuste necesario para adaptarse a la comunidad universitaria; las situaciones de 
vida y aspectos externos; la vida social del estudiante, las relaciones e interacción social, la nostalgia 0 condiciones que depriman al estudiante, el ingreso del estudiante a la fuerza de trabajo, la poca, nula, o deficiente orientación vocacional (p. 167).

Con respecto a la edad de los estudiantes, se evidencia que la mayoría oscila entre 17-20 años, lo cual representa un aspecto positivo para la universidad pues, (Avendaño et al., 2016) afirman que la edad es un factor explicativo del rendimiento académico mencionando que en un mismo curso aquellos alumnos que son más jóvenes obtienen un mejor promedio.

La diferencia entre el género femenino y masculino no es significativa debido a que es relativamente baja, por lo que se puede afirmar que existe equidad de género entre los estudiantes de la ESPAM MFL.

Los resultados revelaron que el $67.7 \%$ de los estudiantes no poseen ningún tipo de seguro que respalde sus vidas y ello supone una alarma hacia las autoridades pues según
(Cámara de Comercio de Quito, 2019) se estima que:

El $80 \%$ de los accidentes que ocurren en los predios de una institución educativa son fracturas en brazos, piernas y cabeza, esto debido a la alta actividad que por naturaleza tienen los estudiantes, ya sea en el recreo o en prácticas académicas o deportivas como partidos de fútbol, basquetbol, entre otros (p. 10).

Es indispensable que los estudiantes tengan la contratación de algún tipo de seguro como una inversión, destinado a salvaguardar al estudiante y permitir que cumpla sin inconvenientes su carrera universitaria. Ante lo mencionado, (Barboza, 2018) señala que: "Existen instituciones privadas y públicas que ofrecen seguros para proteger a sus estudiantes, ya sean de vida, salud o de accidentes que por sus actividades físicas o de laboratorio se requiere de alguna protección mientras los alumnos realizan sus prácticas" (p. 2). Teniendo en cuenta que el respaldo no solo se presenta dentro del aula o universidad, sino también mientras el alumno se dirige a la facultad o sale de ella para regresar a su domicilio. 


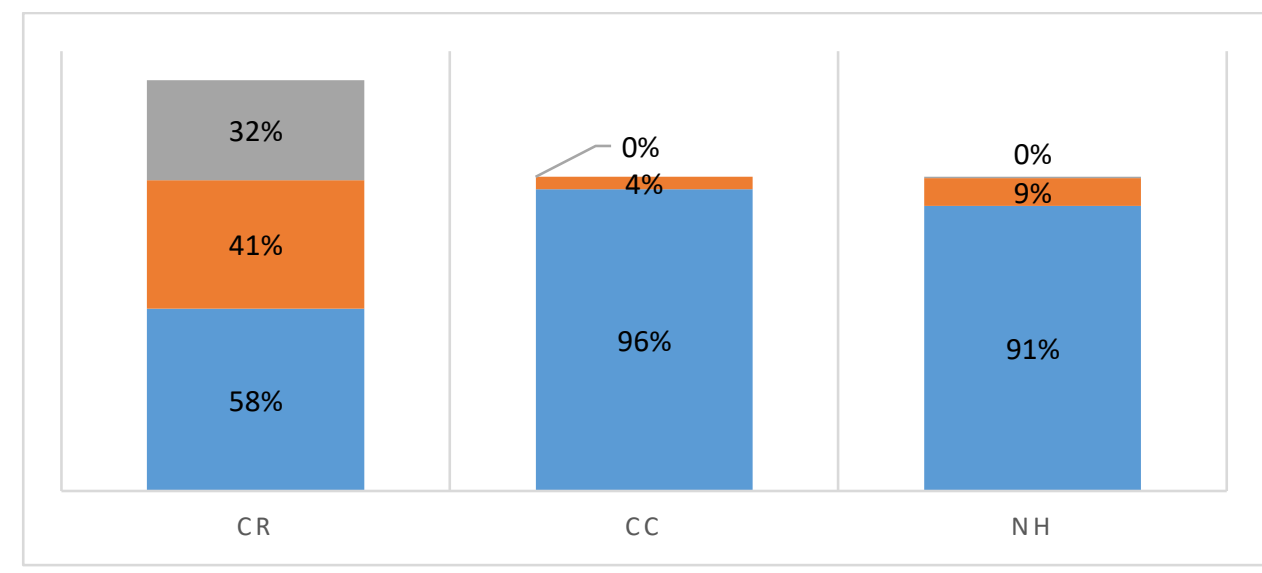

Fig. 2. Datos Sociales. Elaborado por el autor (2020). Nota: CR: cambio de residencia; CC: cambio de carrera; $\mathrm{NH}$ : número de hijos.

En la gráfica de la figura 2, se agruparon las variables consideradas como aspectos sociales de los estudiantes. Se evidencia que la mayoría de los estudiantes no cambiaron su lugar de residencia al ingresar a la universidad es decir el $58.1 \%$, con respecto al $40.9 \%$ que si lo realizó. Iniciar estudios universitarios implica nuevos retos en la vida del estudiante tanto dentro, como fuera de la universidad, algunos requieren mudarse y hacer ajustes en sus rutinas diarias, pues en un universo de masas es necesario encontrar nuevas redes y organizar el tiempo libre (Pérez, 2016).

El $96 \%$ de los estudiantes encuestados afirmaron que no cursaron estudios universitarios previos al ingreso a la ESPAM MFL, ni se cambiaron de institución para cursar la misma carrera, mientras que el $4 \%$ restante si ha realizado y solicitado un cambio de carrera. Ante lo expuesto, (Sancho, 2016), mencionan que el $30 \%$ de los alumnos que acceden a la universidad cambian o abandonan la carrera que eligieron, debido a la falta de orientación en los institutos y el desajuste entre lo estudiado y lo aplicable en el mundo laboral. No obstante, (Ramírez, 2016), señala que 8 de cada 10 estudiantes que ingresaron a una universidad o a una escuela politécnica pública, continuaron sus estudios en primer año. En este contexto, según la Secretaría Nacional de Educación Superior la tasa de retención inicial ha disminuido debido a la implementación del Examen Nacional para la Educación Superior (ENES), ya que, antes el porcentaje 
de deserción era del 52\%; ahora llega al $20 \%$.

El $90.8 \%$ de los estudiantes afirmaron que no tienen hijos, mientras que el $8.8 \%$ tienen entre 1 y 3 hijos y sólo el $0.4 \%$ tiene más de 4 hijos. Los resultados obtenidos son cónsonos con la investigación realizada por (Miller \& Arvizu, 2016), en la cual indica, que al paso del tiempo, la proporción de estudiantes de primer ingreso que llegan a la universidad siendo madres va disminuyendo; en las generaciones analizadas su proporción llega a un $6 \%$ como máximo. Lo que trae las siguientes consecuencias:

Los estudiantes teniendo hijos tienden a realizar rutinas seguidas para cubrir jornadas laborales, escolares y domésticas, distribuyendo su tiempo en una multiplicidad de actividades que llegan a complicarles aspectos como el rendimiento académico, dejar el cuidado de los hijos a terceros, aceptar trabajos que se ajusten a sus horarios 0 incluso suspender sus estudios. Ser padre o madre conlleva a un impacto imprevisto emocional/afectivo, social y económico, y una nueva responsabilidad que afecta la posibilidad de sobrellevar adecuadamente las responsabilidades académicas. Por ende, estos jóvenes tienen un riesgo mayor de deserción universitaria, dadas las consecuencias emocionales, sociales, familiares y económicas que aquello conlleva. (Castañeda, 2015, p. 11)

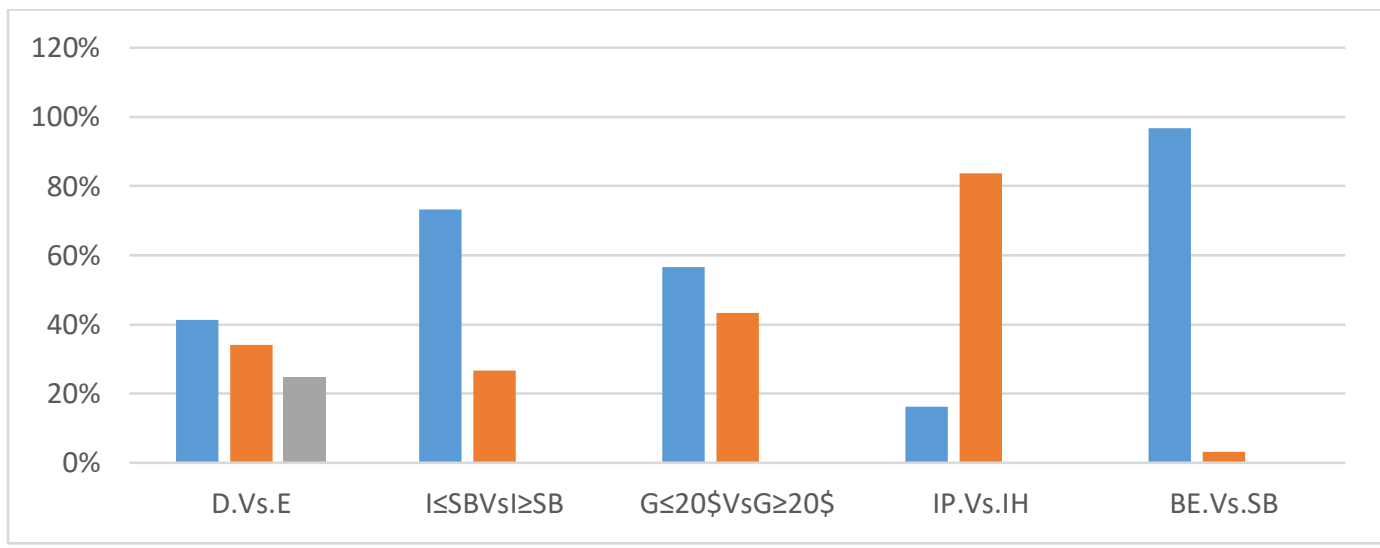

Fig. 3. Datos Económicos. Elaborado por el autor (2020). Nota: D: desempleados; E: empleados; IH: ingresos del hogar; SB: sueldo básico; G: gastos promedios semanales de los estudiantes; IP: ingresos propios (estudiantes que trabajan); BE: estudiantes becados; SB: estudiantes sin beca. 
Con referencia a la pregunta sobre la relación laboral del hogar se obtiene en promedio que el $41 \%$ de los estudiantes señalan que sus madres y padres se encuentran desempleadas; el $34 \%$ indicó que tiene trabajo eventual, mientras que el $25 \%$ indicaron que sus padres tienen negocio propio. Por lo tanto, la encuesta denota que la mayoría de los padres y madres de los estudiantes se encuentran sin empleo. Los resultados coinciden con la Encuesta Nacional de Empleo, Desempleo y Subempleo (INEC, 2019), misma que indica que la tasa de desempleo para las mujeres fue mayor a la de los hombres, a nivel nacional con el $4.64 \%$ de las mujeres en la población económicamente activa estuvieron en situación de desempleo. Sin embrago la tasa de desempleo de los hombres fue de $3.27 \%$. Diversos estudios han demostrado que la relación entre el nivel socioeconómico de la familia y el logro académico, repercute de forma positiva o negativa según el nivel.

Según los datos obtenidos para la variable ingresos del hogar el $73.3 \%$ de los hogares de los estudiantes perciben ingresos menores a un salario básico unificado es decir \$ 394. El $22.7 \%$ oscila entre de $\$ 394$ hasta $\$ 788$ es decir, más de dos salarios básicos. Por otra parte el $3.2 \%$ obtienen ingresos entre $\$ 1182$ y $\$ 1500$ y sólo un $0.8 \%$ generan ingresos de más de $\$ 1500$. Reflejando entonces que, los estudiantes provienen de hogares con un bajo ingreso económico. Por ello, (Mamani Ramos, 2018), argumenta que las familias con bajos niveles socio-económicos son menos propensos a apoyar o alentar a sus hijos a continuar en la educación superior, lo que resulta una desventaja de clase social en relación con el ingreso a la universidad.

En relación a los gastos económicos de los estudiantes semanalmente para acceder a la universidad en promedio el $56.6 \%$ oscila entre $\$ 5$ y $\$ 20$ semanales, el $34.8 \%$ señalaron que utilizan entre $\$ 21$ y $\$ 40$, mientras que $6.6 \%$ indicaron que tienen entre $\$ 41$ y $\$ 60$ dólares de gastos, el $0.6 \%$ gasta entre $\$ 61$ a $\$ 80$ y el restante afirman que sus gastos superan los $\$ 90$ semanal. Los resultados obtenidos son coincidentes con un estudio realizado por estudiantes de la 
Universidad de las Fuerzas Armadas (ESPE) y publicado en la revista digital Argentina 'EF Deportes, el cual señala que en las universidades privadas, el $16 \%$ de los estudiantes tienen gastos superiores a $\$ 5$ diarios solo en materiales escolares. En el caso de las universidades públicas el dato llega al 23.5\%. En este sentido (López de la Madrid et al., 2014), afirma que la mayor parte de los gastos realizados por estudiantes, está destinado a la alimentación, transporte y materiales de estudio, y en los que menos gastan son telefonía fija, actividades de cultura, arte, y deportes, que en su mayoría son gratuitos.

El $83.7 \%$ de los estudiantes indican que no realizan ninguna actividad económica remunerada de manera periódica, mientras que $16.3 \%$ señala que sí. Con respecto a los resultados mencionados (Medranda \& Romero, 2018), aseguran que los problemas familiares repercuten directamente en el rendimiento académico puesto que según su estudio el $25 \%$ indican estar con parejas, en unión de hecho e hijos, y un $15 \%$ tienen que trabajar para poder estudiar y los horarios dispersos de las universidades también les impiden estudiar todas las asignaturas del nivel; el otro $60 \%$ son hijos de familia que dependen de sus padres y de los recursos económicos que ellos les pueden brindar para cumplir con sus actividades estudiantiles, este grupo indicó que el 35\% tienen problemas familiares que dificultan un normal rendimiento económico.

La mayor parte de los estudiantes no tienen una beca concedida por el Estado, asi lo demuestra la encuesta realizada donde el $96.8 \%$ manifestaron no tener el beneficio de becas de estipendio; el $2.9 \%$ restante mantienen becas asignadas por su situación de vulnerabilidad socioeconómica. Es importante destacar que los estudiantes no están informados de las opciones y posibilidades que tienen para aplicar a una beca de asignación estatal, a pesar que en la Ley Orgánica de Educación Superior (Asamblea Nacional, 2018), en su Art. 183, establece que son funciones de la Secretaria de Educación Superior, Ciencia, Tecnología e Innovación: "Ejercer la rectoría de las políticas públicas en el ámbito de su competencia" y "Diseñar, administrar e instrumentar la política de becas 
del gobierno para la educación superior ecuatoriana (...)" entre otras.

La beca es un incentivo económico que motiva al estudiante a seguir mejorando y por ende a culminar sus estudios. Los datos de las encuestas realizadas dieron como resultado que los estudiantes de los primeros semestres coinciden en un $97.4 \%$ equivalente a 900 estudiantes que no tiene una beca emitida por la universidad; sin embargo solo $1.6 \%$ equivale a 15 estudiantes que la universidad les otorga beca por rendimiento académico; mientras que el $0.4 \%$ tiene beca por escasos recursos; y apenas el $0.3 \%$ tiene beca por ayuda económica otorgada por la universidad; así mismo solo el $0.2 \%$ tiene beca por discapacidad. Estos datos muestran la importancia que la universidad debe considerar al otorgar becas a los estudiantes con recursos económicos bajos.

\section{Discusión}

"El rendimiento académico es un conjunto de factores que inciden en el aprendizaje del estudiante y en el logro obtenido, sea de forma inmediata, medido por notas, o de forma mediata, vista en el desempeño profesional" (TorresDíaz et al., 2016, p. 45). Razón por la cual, las instituciones de educación superior están llevando a cabo diversas acciones para apoyar la mejora del rendimiento académico y evitar la deserción de los estudiantes.

Durante el proceso educativo, el estudiante se encuentra inmerso en varios factores, que contribuyen al logro de su titulación; el rendimiento académico es fundamental, ya que está comprendido por un conjunto de factores que inciden en el desempeño y aprendizaje del estudiante, el cual es abordado desde diferentes perspectivas, como su efectividad y el nivel de éxito en cumplimiento de objetivos, ya que el mismo puede generar varias problemáticas como abandono, rezago, y reprobación.

De acuerdo con el modelo educativo de la (ESPAM MFL, 2016), señala que: "El perfil es un conjunto de capacidades que identifican la formación de una persona para asumir las condiciones óptimas, las responsabilidades propias del desarrollo de funciones $y$ tareas de una determinada profesión" (p. 12). 
Este perfil académico determina: "Las competencias del egresado para lograr el desempeño laboral según su área disciplinar; son las cualidades que demuestra para resolver problemas en un entorno de trabajo cambiante e incierto, y la capacidad para aprender y afrontar nuevas situaciones" (Martelo et al., 2017, p. 16)

El perfil académico 0 perfil profesional como muchos autores lo describen, son aquellos requerimientos que debe poseer 0 tener un estudiante como: conocimientos, habilidades, valores y actitudes; es decir, un conjunto de capacidades y aptitudes que identifican la formación de una persona para asumir cada una de las responsabilidades que se le asigna, formando así un perfil profesional eficiente y eficaz, capaz de resolver y afrontar diferentes situaciones que se le presenten.

En referencia al nivel socioeconómico, (Olmeda, 2016) plantea: "que es el conjunto de características cuantitativas y cualitativas, es un término que hará referencia a todas las personas de una misma vivienda, de modo que un sujeto "hereda" estatus socio-económico del hogar, no el de sus miembros por separado" (p. 123). El mismo que, de acuerdo a (Coschiza et al., 2016): comprende una serie de "características del hogar asociadas con la capacidad de consumo del grupo familiar y que no debe confundirse 0 identificarse directamente con la pertenencia a una clase social determinada" ( $p$. 34). De esta manera con base a los rasgos cualitativos y cuantitativos se pueden realizar análisis a partir de la creación de categorías basadas en el consumo familiar.

Siendo el nivel socio-económico uno de los problemas de los que más carecen los estudiantes universitarios, pues éste asegura 0 descarta su permanencia. El nivel socio-económico comprende las características cuantitativas y cualitativas asociadas a un individuo, así como la capacidad de consumo que tiene una vivienda u hogar; sin embargo, ésta no debe asociarse con la pertenencia a una clase social determinada; las características socio-económicas de un estudiante universitario se refieren a aspectos como: los ingresos familiares, el nivel económico y nivel de estudios de los padres. 


\section{Conclusiones}

La cantidad de estudiantes que cursan el segundo nivel es inferior a los de primero con una diferencia del $31 \%$, lo que significa que ese porcentaje de estudiantes no aprueban el primer nivel. A este hecho se le atribuye como causa principal la deserción estudiantil. El $67.7 \%$ de los estudiantes no cuentan con ningún tipo de seguro que respalde sus vidas, tanto dentro de la universidad como fuera de ella en el trayecto para regresar a sus domicilios.

Con respecto a las variables relacionadas con aspectos sociales, la mayoría de los estudiantes no realizaron cambio de residencia al ingresar a la universidad con un $58.1 \%$; sin embargo, existe una proporción del $40.9 \%$ que si cambió su lugar de residencia, y que de éstos el $31.8 \%$ arrienda un departamento, ya que debido al cambio requieren mudarse y hacer ajustes en sus rutinas diarias; asimismo, del total de los encuestados el $90.8 \%$ de los estudiantes no tienen hijos, sin embargo, el $8.8 \%$ que corresponde a 81 estudiantes tienen de 1 a 3 hijos, siendo este un limitante, que aunque no es un número elevado si existen estudiantes con hijos, y con el paso del tiempo, la proporción de estos estudiantes que llegan a la universidad siendo padres o madres va disminuyendo ya que realizan otras actividades que repercuten directamente en el rendimiento académico, e incluso algunos llegan a suspender sus estudios universitarios.

Los hallazgos derivados de las variables económica son de gran interés y relevancia, pues la mayoría de los estudiantes dependen económicamente de sus padres, lo que resulta de gran preocupación ya que en promedio el $41 \%$ de los padres de familia están desempleados y el $34 \%$ de ellos cuentan con un empleo de manera eventual y el $21 \%$ tienen un empleo formal o negocio propio; lo que pone en evidencia que el $73.3 \%$ de los estudiantes provienen de hogares con bajos recursos económicos, por lo tanto la probabilidad de que aumenten las tasas de deserción y disminuya el rendimiento académico es bastante alta. 


\section{Bibliografía}

Asamblea Nacional. (2018). ECUADOR Asamblea Nacional Ley Orgánica de Educación Superior (LOES) (Oficio No. T. 4454-SNJ-101512).

https://siteal.iiep.unesco.org/s ites/default/files/sit_accion_fil es/ec_6011.pdf

Asamblea Nacional de la Republica del Ecuador. (2008). CONSTITUCION DE LA REPUBLICA DEL ECUADOR 2008 Decreto Legislativo 0 Registro Oficial. In Constitución del Ecuador. www.lexis.com.ec

Avendaño, C. A., Gutiérrez, K. A., Salgado, C. F., \& Alonso-DosSantos, M. (2016). Rendimiento académico en estudiantes de ingeniería comercial: Modelo por competencias y factores de influencia.

Formacion Universitaria, 9(3), 3-10. https://doi.org/10.4067/S0718 $-50062016000300002$

Barboza, G. (2018, October 31). ¿Cómo son los seguros de vida para universitarios? https://www.rastreator.mx/seg uros-de-vida/articulosdestacados/seguros-paraestudiantes

Cámara de Comercio de Quito. (2019, September 25). Beneficios del Seguro Estudiantil y cómo utilizarlo con Seguros de Vida, Salud y Accidentes Personales de AIG-Metropolitana Cía. de Seguros y Reaseguros S.A. https://ccq.ec/beneficios-delseguro-estudiantil-y-comoutilizarlo-con-seguros-devida-salud-y-accidentespersonales-de-aigmetropolitana-cia-deseguros-y-reaseguros-s-a/

Castañeda, M. (2015). Ser Estudiantes, Madres y Padres: Una dualidad cotidiana [Universidad de Chile].

http://repositorio.uchile.cl/bitst ream/handle/2250/135041/M emoria de Titulo Maternidad y Paternidad Universitaria FINAL.pdf?sequence $=1$

CEPAL. (2019, November 15). Educación | Comisión Económica para América Latina y el Caribe. https://www.cepal.org/es/tem as/educacion

Coschiza, E., Cristóbal, C., Martín, J., \& Gapel, G. (2016). Características

Socioeconómicas

Rendimiento Académico. EI Caso de una Universidad Argentina. REICE. Revista Iberoamericana Sobre Calidad, Eficacia y Cambio En Educación, 14(3), 51-76. https://doi.org/10.15366/reice 2016.14.3.003

ESPAM MFL. (2016). Modelo Educativo Escuela Superior 
Politécnica Agropecuaria de Manabí "Manuel Félix López (Escuela Superior Politécnica Agropecuaria de Manabí "Manuel Félix López" (ed.)). Editorial Humus. http://www.espam.edu.ec/rec ursos/sitio/espam/ModeloEdu cativo2016.pdf

Hernández, R., Fernández, C., \& Baptista, P. (2014). Metodología de la investigación (P. Baptista (ed.); 6th ed.). McGRAWHILL.

http://observatorio.epacartag ena.gov.co/wpcontent/uploads/2017/08/met odologia-de-la-investigacionsextaedicion.compressed.pdf

INEC. (2019). ENEMDU. https://www.ecuadorencifras. gob.ec/enemdu-diciembre2019/

INEVAL. (2020). Instituto Nacional de Evaluación Educativa | Página oficial del Instituto Nacional de Evaluación Educativa. Instituto Nacional de Evaluación Educativa. http://www.evaluacion.gob.ec

López de la Madrid, M. C., De los Monteros Cárdenas, A. E., Rojo Morales, D., Flores Guerrero, K., \& Rojas García, A. (2014). Hábitos de consumo del estudiante universitario. El caso del Centro Universitario del Sur, de la Universidad de Guadalajara. Nova Scientia, 7(13), 352. https://doi.org/10.21640/ns.v7 i13.8

Mamani Ramos, O. J. (2018). Factores que influyen en la elección de una universidad en los estudiantes jóvenes y adultos del Perú. 1-70. https://eprints.ucm.es/id/eprin t/49860/1/TFM_Sept2018_M amami_E-prints.pdf

Martelo, R. J., Villabona, N., \& Jiménez-Pitre, I. (2017). Guía Metodológica para Definir el Perfil Profesional de Programas Académicos Guía Metodológica para Definir el Perfil Profesional de Programas Académicos Mediante la Herramienta Ábaco de Régnier Methodological Guide to Define the Professional Profile of Academic Programs by using the Regnier Abacus. 10(1), 15-24. https://doi.org/10.4067/S0718 $-50062017000100003$

Medranda, R., \& Romero, S. (2018). Problemas familiares y el rendimiento académico. Caribeña de Ciencias Sociales, mayo. https://www.eumed.net/rev/ca ribe/2018/05/estudiantestrabajo-social.html

Miller, D., \& Arvizu, V. (2016). Ser madre y estudiante. Una exploración de las 
características de las universitarias con hijos $y$ breves notas para su estudio. Revista de La Educación Superior, 45(177), 17-42. https://doi.org/10.1016/J.RES U.2016.04.003

Olmeda, L. (2016). Nivel Socioeconómico

$Y$

Rendimiento Académico:

Estudiantes Resilientes

[Universidad Complutense de Madrid].

https://core.ac.uk/download/p df/78501244.pdf

Palencia Avendaño, M. L. (2012). Metodología de la Investigación. In Educación para todos: Vol. Módulo 1 (UNAD, p. 205). https://www.academia.edu/26 669099/Metodología_de_la_I nvestigación_MODULO_1_M ETODOLOGÍA DE LA INVE STIGACIÓN_MÓDULO_Elab orado por MARÍA LUISA P ALENCIA_AVENDAÑO_Meto dología_de_la_Investigación MODULO_2

Peña, A. (2017). ¿La experiencia Universitaria influye en el abandono de la Universidad? INNOVA Research Journal, 2(10), 162-168. https://doi.org/10.33890/innov a.v2.n10.2017.531

Pérez, I. (2016). El Proceso De Adaptación De Los Estudiantes a La Universidad En El Centro Universitario De Los Altos De La Universidad
De Guadalajara. Repositorio Institucional Del Instituto Tecnológico y de Estudios Superiores de Occidente, 297. https://rei.iteso.mx/handle/11 $117 / 3591$

Ramírez, R. (2016). Universidad urgente para una sociedad emancipada. Chasqui. Revista Latinoamericana de Comunicación, 0(131), 1580.

https://doi.org/10.16921/CHA SQUI.V0I131.2897

Sancho, M. (2016). El 30\% de los universitarios cambia 0 abandona la carrera. Hacer Familia.

https://www.hacerfamilia.com /jovenes/noticia-30universitarios-cambiaabandona-carrera20160107133728.html

Torres-Díaz, J. C., Duart, J. M., Gómez-Alvarado, H. F., Marín-Gutiérrez, I., \& Segarra-Faggioni, V. (2016). Internet use and academic success in university students. Comunicar, 24(48), 61-70. https://doi.org/10.3916/C482016-06 